\title{
Design and Implement of Adaptive Variable Rate Communication System Based on Soc And Rfic
}

\author{
Dong Limin, Liu Yuan, Kong Qinglei \\ Harbin Institute Of Technology \\ 1010510507@163.Com,Dr.Yuanliu@Gmail.Com,Hit_Kongqinglei@163.Com
}

\begin{abstract}
An adaptive variable-rate inter-satellite communication method based on SOC and RFIC was proposed, where different communication rates were selected for variable inter-satellite distance. Our method alerted the length of the signal through coding way to adjust the sending rate and regulated the receiving rate according to the sending rate when the inter-satellite distance changed, realizing the unity of communication reliability and validity. Based on SOC and RFIC, the variable-rate communication system was implemented, and results of the tests and experiments on this system were given to illustrate the proposed method.
\end{abstract}

Keywords-SOC, RFIC, Adaptive, Variable rate communication

\section{INTRODUCTION}

Satellites formation flight was a new field right now, which could carry out space missions instead of a complex one. This technology could enhance the anti-destruction and anti- jamming ability [1], but it also put forward new requirements for the weight, the volume, the function density and the power of the satellites [2]. So the communication system used in the satellites formation flight must be small, light, and high function density [3].

At the present time, two kinds of method were proposed: one was variable communication method, the other was $f$ spread spectrum communication method [4,5]. Spread spectrum communication which needed complex devices to process the base band signal would be restricted in the small satellite. Variable communication method was reported infrequently, except the SURREY satellites communication method which varied the length of the communication frame by the distance [6]. According to the need of the miniaturization, reliability and the efficiency of the communication system that needed different communication rate for variable inter-satellite distances, a new structure of communication system based on SOC and RFIC was established, which could ensure the reliability and the efficiency of the communication whether the distances were near or far.

\section{DESIGN OF VARIABLE RATE INTER-SATELLITE}

\section{COMMUNICATION SYSTEM}

\section{A. Analysis of inter-satellite link}

On the process of the satellite formation flight, intersatellite distances could be variable from several decameters to hundreds kilometers when the formation order reconstructed with the mission. In the far distance, the information need to exchange only included the distance, the velocity, the position, the attitude and some instruction, In the near distance, image data might be transmit result in huge data transmission, which was a feature of the formation flight communication [7].

The relationship of the emission power, communication distance and bit error rate was restricted by the communication link function.

$$
E_{b} / N_{0}=10 \lg P_{\mathrm{T}}+10 \lg \left(\frac{L_{1} G_{\mathrm{T}} L_{a} G_{r}}{k T_{s}}\right)-10 \lg R-32.44-20 \log d-20 \log f
$$

As shown above, when the communication distance increased, we had to increase the emission power or decrease the communication rate to ensure the $\mathrm{E}_{\mathrm{b}} / \mathrm{N}_{0}$ of the receiving end unabated to ensure the reliability of the communication. Increasing the emission power required more power supply so that it was not a reasonable method when the power supply was restricted. Decreasing the communication rate could be used to increase the SNR.

\section{B. Design of the hardware}

The main function of the communication system contained receiving, demodulation, dealing with base band data, modulation and sending. The hardware of our variable communication system consisted of SOC processor, RFIC and the antenna, which was shown in Figure 1.

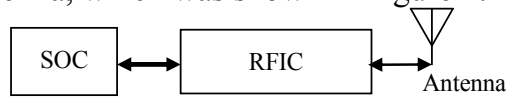

Figure 1 Function diagram of communication system

We chose C8051F040 made by Cygnal company as our SOC processor, whose speed was greatly increased compared with the standard 8051 processor. C8051F040 was used to control the RFIC such as initialization, configuration, sending and receiving data. We could configure the RFIC to work in different frequency to realize FDMA way of working, or control the timing order to realize TDMA way of working.

We chose nRF2401 as RFIC, which had two effective modes: burst mode and direct mode. The burst technology uses on-chip FIFO to clock in data at a low data rate and transmit at a very high rate thus enabling extremely power reduction. In the direct mode, the data date lied on the processor sending and receiving rate. The direct mode was used in the variable communication system.

\section{Method of variable communication}

Variable rate communication technology depended on the repetitions was implemented by the coding and decoding of the duplication code. 
As the Figure 2 shown, on the sending terminal the data could be coding as the duplication code. The repetitions N0 could be computed as the communication rate dividing the highest code rate of the RFIC. One bit of the date could be modulated and sent for N0 times, and then the next bit was processed equally, until the whole frame data was sent completely.

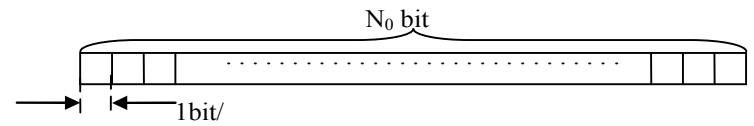

Figure 2 Coding in the sending end

As the Figure 3 shown, on the receiving terminal, the date received need to be demodulated, sampled, bit synchronized, adjudged, outputted, frame synchronized and processed to accomplish the communication.

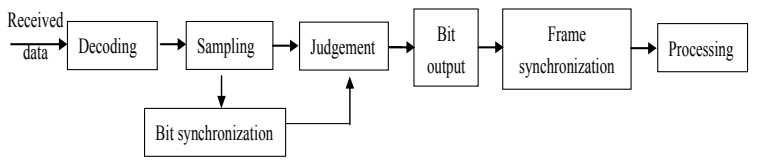

Figure 3 Decoding principle of the receiving end

The decoding was accomplished automatically by nRF401, and the other steps must be implemented by the SOC processor. The sampling module could sample the data demodulated in a fixed fast cycle, then store the data sampled in the ram.

Bit synchronizing module could extract the clock synchronization according to the data stored in the ram. The diagram was shown as Figure 4.

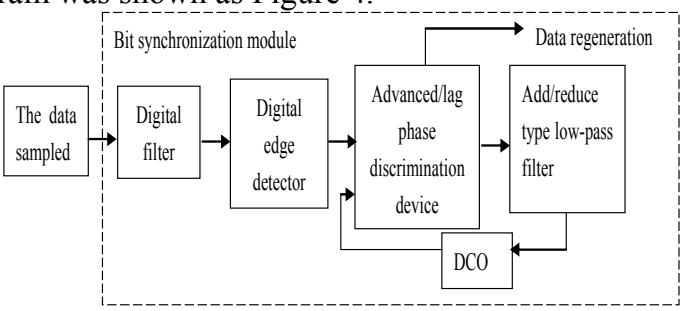

Figure 4. Principle of bit synchronization module

In the bit synchronization, the data after sampling would be filtered to remove burr brought by the noise, then the 1-0 jumping edges of the data filtered would be extracted as the clock synchronized in the coming data. And the extracted edge would be input into digital phase-locked loop to detect the phase with local clock, and then adjusted the local clock frequency to make it synchronize with the edge of the coming data. The local clock was used for data regeneration. Local DCO was controlled by software, whose working frequency could be synchronized in input data to realized variable rate communication.

In one clock cycle, the judgment module judged the data sampled according to the sum of the data. This module could reduce the impact of noise to improve receiving reliability.

Frame synchronization and signal process module would compose the bit streams into a whole communication frame according to the format.
In the communication process, the sending end must send $0-1$ codes before the communication frames. Receiving end complete bit synchronization according to the sending codes.

\section{REALIZATION AND VALIDATION OF VARIABLE RATE}

\section{COMMUNICATION SYSTEM}

\section{A. Realization of variable rate communication system}

When the variable rate communication system applied to star communications, ciculator, low noise amplifier and power amplifier was needed to enhance communication ability. The communication system diagram was shown in figure 5 .

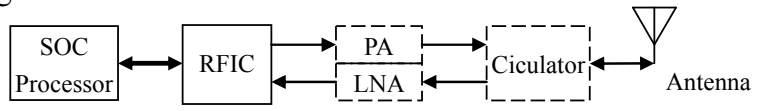

Figure 5. Consist of communication system

Ciculator was mainly used to isolate the sending signal and receiving signal to realize the system half-duplex work.

The LNA was used to enhance the receiving ability whose working band of for 2.4-2.5 GHz, Receive signals via LNA amplification, were sending to the isolating amplifier level, then sending to the receive unit for signal processing.

PA was used to enhance the sending ability, whose output eventually through the ciculator, was sent to the antenna .

Circuit board of variable rate communication system was shown as figure 6 .

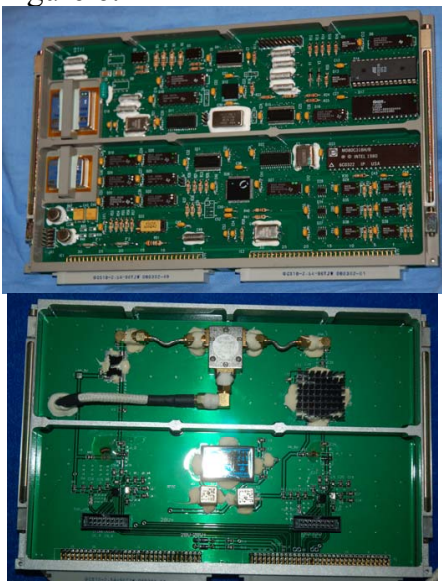

Figure 6. Circuit board of variable rate communication system

Variable rate communication algorithm in software would be realized by $\mathrm{C} 8051$.

First by software timer interrupt way we would finish data sampling, then collected the data in the storage for the processing;

After quit of the interrupt, filter remove the burr of the signal through the way of digital, then store the filtered data into the new storage area.

The filtered data would be differential encoded to jump to get the jumping edges of the data, and adjusted current 
clock frequency according the phase relationship with the jumping edge to make the new clock lock in the input signal and adapt to new data rate. And the output bits of data were also locked in the input signal.

Finally, data was processed according to the predetermined frame format to complete the communication.

\section{B. Validation of variable rate communication system}

Validation method of variable rate communication system was shown as figure 7 .

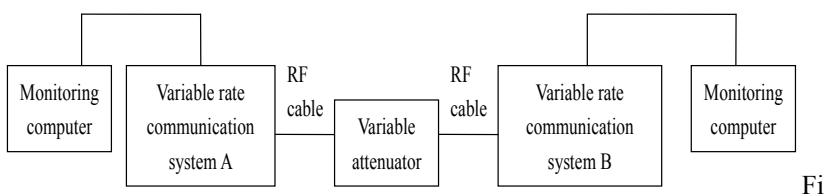

gure 7. Validation mathod of variable rate communication system

Validation system consisted of two variable rate communication systems, monitoring computer, variable attenuator and high frequency cable. A was used as the sender, and B was used as the receiver, variable attenuation was used to adjust the attenuation of the link. The monitoring computer communicated with the C8051 through a serial port to control the communication rate, and receive the status information.

In the experiment of the validation, $\mathrm{A}$ and $\mathrm{B}$ first adopts high code rate. A sent 1-0 codes first, and B static bit error rate according to the received 1-0 codes .Then gradually changed the value of the variable attenuator. When the bit error rate was more than 10-3, the communication rate would be changed through monitoring computer $\mathrm{A}$, then monitor computer $\mathrm{B}$ to adapt its communication rate, and continue to static bit error rate.

During the experiment, the monitoring terminal use the serial port debugger to control communication system and monitor the communication process data and the state.

(1) Validation of adaptive variable rate communication algorithm

The sending end sent commands which adapted the communication rate of the sending end through a serial port debugger, which was shown in figure 8 .

From the current sending rate and the previous sending rate can be seen, after the command of the sending end, the sending rate changed to the setting rate.

Communication rate and lock status of the receiving end could be monitored through a serial port as shown in figure 8.

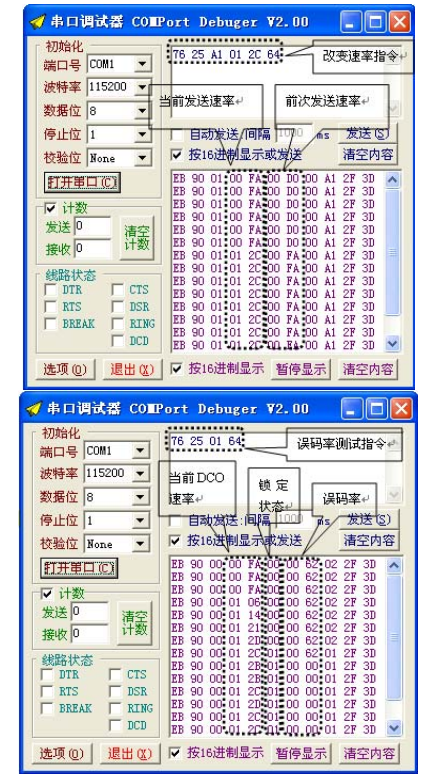

Figure 8 . The communication of sending and receiving end(L:sending

$$
\text { end;R:Receiving end) }
$$

As could be seen, the communication rate of the receiving end changed according to the sending end, and DCO rate of the receiving end was adjusted to the sending end.

(2) Validation of communication rate and error bit rate

As figure 9 shown, we would calibrate the high frequency cable and attenuator before the experiment: adjusted the attenuation of variable attenuator to 0 , and then the standard source sent $0 \mathrm{dBm}$ standard signal., then We could get the power line attenuation quantity by reading the signal on the power meter

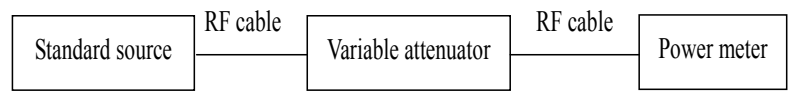

Figure 9. Adjusting attenuator and cable

After calibration, variable rate communication system of the sending end sent fixed $0-1$ codes, in different communication rate the bit error rate would be test when the receiving end locked in the sending end. As shown in figure 9, bit error rate test command would be sent by the monitoring computer, the receiving end would automatically clear the statistics result before and then statistic the bit error rate after the received instructions to get the table 1 .

As we known, attenuation was proportional to quantity of communication distance. From analysis of table 1, with the same attenuation, reliability would be better as the communication rate was lower, and the lowering communication rate was effective to ensure the reliability of communication. We could get the conclusion that variable rate communication could realize the reliable and efficient communication in different distances. 


\section{CONCLUSIONS}

Inter-satellite communication for satellites formation flight was a complex and difficult engineering for its high reliability and efficiency request. Satellite high-speed motion and complex space environment made satellite communications be different from ground communication. Aiming at the small satellite formation flight communication problems, we proposed a variable rate communication system based on SOC and RFIC to solve problem the reliability and efficiency of communication when distance varied, with the characteristics of miniaturization, communication rate adaptive. A strict verification about the communication system was implemented The variable rate communication system had a certain practical innovation, and lay a foundation for the next satellite formation flight research work.

\section{REFERENCES}

[1] Dr Kim Luu; Maurice Martin; etc.. University nanosatellite distributed satellite capabilities to support techsat21. 13th AIAA/USU Small Satellite Conference. 1999,8, PP1 9
[2] Kitts; Christopher; Twiqqs; Robbert; etc.. Emerald-A Low-cost Spacecraft Mission for Validating Formation Flying Technologies. IEEE Aerospace Applications Conference Proceedings. 1999,2, PP:217 226

[3] Xu Guodong; Tan Xiaojun. Design of electronic system in high spectrum imaging system. National defense science report. 1996, 9, PP:1 43

[4] Wang Shilian, Lu Jun, Li Haichuan, Zhang Eryang. Research on inter-satellite communication protocol . Space craft T\&TC transaction. 2002, 4, PP:53 57

[5] S. Horan and B. Anderson. Three corner at Constellation-New Mexico Inter-satellite University: Communications, and Ground Stations and Network. 13th AIAA/USU Conference on Small Satellites, 1999,8:1 9

[6] H.Tiggeler, T.Vladimimirova, D.zheng. Experiences Designing a System-on-a-Chip for Small Satellite Data Processing and Contral. Proceeding of 4th Military and Aerospace Application of Programmable Devices and Technologies International Conference. 2000,9:1 11

[7] Goodman, Nathan A, Stiles,James M. Synthetic aperture characterization of radar satellite constellations. International Geoscience and Remote Sensing Sympoium(IGARSS). 2002:665 667

TABLE 1. Bit ERror RATE of VARIOUS COMMUNICATION RATE

\begin{tabular}{ccccc} 
attenuation $(\mathrm{db})$ & Communication rate(bps) & $\mathrm{N} 0$ & $\mathrm{~Eb} / \mathrm{N} 0$ & $\mathrm{BER}$ \\
\hline 90 & 1000 & -41.6 & 12.2 & 0 \\
\hline 90 & 1200 & -41.6 & 11.2 & 0 \\
\hline 90 & 1600 & -41.6 & 10.2 & 0 \\
\hline 90 & 2000 & -41.6 & 9.2 & $5.5 \mathrm{E}-5$ \\
\hline 90 & 2400 & -41.6 & 8.2 & $2.7 \mathrm{E}-4$ \\
\hline 90 & 3200 & -41.6 & 7.2 & $1.0 \mathrm{E}-3$
\end{tabular}

